PROCEEDINGS OF THE

AMERICAN MATHEMATICAL SOCIETY

Volume 104, Number 3, November 1988

\title{
CRITICAL VALUES OF FREDHOLM MAPS
}

\author{
P. T. CHURCH AND J. G. TIMOURIAN
}

(Communicated by Haynes R. Miller)

\begin{abstract}
For a $C^{r}$ Fredholm map $A: X \rightarrow Y$, let $T_{k}(A)$ be the set of $x \in X$ such that codim Range $D A(x) \geq k$. The homotopy of $Y-A\left(T_{k}(A)\right)$ is related to that of $Y$, examples are given, and a factorization result is proved.
\end{abstract}

THEOREM 1. Let $X$ and $Y$ be $C^{r}$ manifolds modeled on (real) Banach spaces, $X$ separable and $Y$ metrizable, let $A: X \rightarrow Y$ be a $C^{r}$ Fredholm map of index $\nu$, and let $T_{k}(A)$ be the set of $x \in X$ such that codim Range $D A(x) \geq k \quad(k=0,1, \ldots)$. Then the homomorphism

$$
i_{*}: \pi_{m}\left(Y-A\left(T_{k}(A)\right), y_{0}\right) \rightarrow \pi_{m}\left(Y, y_{0}\right)
$$

on the $m$ th homotopy groups $(m=0,1, \ldots)$ induced by inclusion $i$ is

(a) an isomorphism (onto) for $m+2 \leq k$ and $r=1+\max (\nu+m+1,0)$, and is

(b) onto for $m+1 \leq k$ and $r=1+\max (\nu+m, 0)$.

Differentiable $\left(C^{r}\right)$ Fredholm maps (Definition 2), defined by Smale [Sm], are useful in the study of certain nonlinear elliptic boundary value problems. Assuming $r>\max (\nu, 0)$ he proved the Smale-Sard Theorem [Sm, p. 862, (1.3)]: the set of critical (or singular) values $A\left(T_{1}(A)\right)$ is meager in $Y$; for non-Fredholm maps this conclusion need not be true, even if $r=\infty[\mathbf{K}]$. Since a Fredholm map is locally proper [Sm, p. 862, (1.6)], the Smale-Sard Theorem is a special case of Theorem 1, viz. case (b) with $m=0$ and $k=1$.

The analog of Theorem 1 for singular homology with integer coefficients follows from the Whitehead Theorem [Sp, p. 399, Theorem 9]. For the finite dimensional case Theorem 1 is given in [C-4, p. 1035, Theorem 1]. The differential hypotheses are best possible (Example 5) if $k=m+2$ in case (a) and $k=m+1$ in case (b). By [CT-2, (1.5)b)] (and local properness [Sm, p. 862, (1.6)]) Theorem 1, case (a) with $m=0$ and $k=2$ is [Mi, p. 291, Theorem A], except that for $\nu<0$ our hypothesis is $C^{1}$, while she requires $C^{2}$. In case $m=0$ isomorphism in Theorem 1 means bijection.

DEFINITION 2. If $E$ and $F$ are Banach spaces, a bounded linear transformation $\Phi: E \rightarrow F$ is Fredholm if (i) $\operatorname{dim} \operatorname{ker} \Phi<\infty$, (ii) Range $\Phi$ is closed in $F$, and (iii) codim Range $\Phi<\infty$; index $\Phi$ is defined to be $\operatorname{dim} \operatorname{ker} \Phi$ - codim Range $\Phi$. A $C^{1}$ map $A: X \rightarrow Y$ on $C^{1}$ Banach manifolds is Fredholm if $D A(x)$ is Fredholm for each $x \in X$. If $X$ is connected, then index $D A(x)$ is independent of $X$ and is called index $A$; if $X$ is not connected, we assume that index $D A(x)$ is independent

Received by the editors August 7, 1987.

1980 Mathematics Subject Classification (1985 Revision). Primary 58C27.

Key words and phrases. Fredholm maps, singularities of differentiable maps, homotopy groups. The authors were partially supported by NSERC Contract A7357. 
of component of $X$. The singular set, or critical set, $S A$ is $T_{1}(A)$, the set of $x \in X$ for which $D A(x)$ is not surjective.

LEMMA 3. Let $(\Delta, \Gamma)$ be a finite polyhedral pair such that $\Delta-\Gamma$ is a $t$-manifold (without boundary), let $Y$ be a $C^{r} \quad(r=1,2, \ldots)$ manifold with metric $d$ modeled on a Banach space $E$, let $\gamma: \Delta \rightarrow Y$ be $C^{0}$, and let $\rho: \Delta \rightarrow[0, \infty)$ be $C^{0}$ and 0 precisely on $\Gamma$. Then there exists a $C^{0}$ map $\delta: \Delta \rightarrow Y$ such that $\delta \mid(\Delta-\Gamma)$ is $C^{r}$ and $d(\gamma(x), \delta(x)) \leq \rho(x)$ (thus $\delta|\Gamma=\gamma| \Gamma)$.

In the following proof int $N$ will refer to $\left(\operatorname{int}_{\Delta} N\right) \cap(\Delta-\Gamma)=\operatorname{int}_{\Delta-\Gamma} N$, while bdy $\mathrm{N}$ will refer to $\mathrm{Cl}_{\Delta} N-\operatorname{int} N$.

PROOF. We may suppose that $\Gamma=\operatorname{bdy}(\Delta-\Gamma)$. There are a finite number of charts $\phi_{i}: U_{i} \rightarrow E(i=1,2, \ldots, n)$ such that $\gamma(\Delta) \subset \bigcup_{i} U_{i}$. We may suppose that $\phi_{i}\left(U_{i}\right)$ is convex. Let $\eta>0$ be the Lebesgue number of the open cover $\gamma^{-1}\left(U_{i}\right)$ of $\Delta$, give $\Delta$ a subdivision with mesh less than $\eta / 2$, and let $\Delta_{i}$ be the union of the closed $t$-simplices $\sigma \subset \gamma^{-1}\left(U_{i}\right)$; then $\Delta_{i}$ is a finite polyhedron, $\gamma\left(\Delta_{i}\right) \subset U_{i}$, and $\Delta=\bigcup_{i}$ int $_{\Delta} \Delta_{i}$. Let $\varepsilon>0$ be the minimum of $d\left(\gamma\left(\Delta_{k}\right), Y-U_{k}\right)(k=1,2, \ldots, n)$, and define $\rho_{i}: \Delta \rightarrow[0, \infty)$ to be a $C^{0}$ map such that $\rho_{i}(x)>0$ if and only if $x \in \operatorname{int} \Delta_{i}$ and $\rho_{i}(x) \leq \min (\varepsilon, \rho(x)) / n$.

It suffices to define inductively $C^{0}$ maps $\delta_{i}: \Delta \rightarrow Y \quad(i=0,1, \ldots, n)$ such that $\delta_{0}=\gamma, \delta_{i}$ is $C^{r}$ on $\Omega_{i}=\bigcup_{k \leq i}$ int $\Delta_{k}$ and $d\left(\delta_{i}(x), \delta_{i-1}(x)\right) \leq \rho_{i}(x)$ for $i>0$ and all $x \in \Delta$. Then $\delta_{n}$ will be the desired function $\delta$.

Suppose that $\delta_{0}, \delta_{1}, \ldots, \delta_{i-1}$ have been defined $(i>0)$; we now define $\delta_{i}$. There exists a $C^{0}$ map $\tau_{i}: \Delta_{i} \rightarrow[0, \infty)$ such that $\tau_{i}(x)=0$ if and only if $x \in$ bdy $\Delta_{i}$ and

(1) if $x \in \Delta_{i}$ and $y \in U_{i}$ with $\left\|\phi_{i}\left(\delta_{i-1}(x)\right)-\phi_{i}(y)\right\| \leq \tau_{i}(x)$, then $d\left(\delta_{i-1}(x), y\right) \leq$ $\rho_{i}(x)$. For each $x \in$ int $\Delta_{i}$, choose an open neighborhood $V$ of $x$ such that

(2) diam $(\bar{V})<(1 / 2) d\left(\bar{V}\right.$, bdy $\left.\Delta_{i}\right)$ (thus $\bar{V} \subset \operatorname{int} \Delta_{i}$ ) and,

(3) if $y, z \in \bar{V}$, then $\left\|\phi_{i}\left(\delta_{i-1}(y)\right)-\phi_{i}\left(\delta_{i-1}(z)\right)\right\|<\min \left\{\tau_{i}(w): w \in \bar{V}\right\}$. The $V$ constitute an open cover of int $\Delta_{i}$; let $W_{j}(j=1,2, \ldots)$ be a countable locally finite refinement, and let $W_{0}=\Omega_{i-1} \cap$ int $\Delta_{i}$. Let $\psi_{j}$ be a $C^{\infty}$ partition of unity subordinate to the cover $W_{j}(j=0,1, \ldots)$; we may suppose that $\phi \neq W_{j} \not \subset W_{0}$ for $j>0$, so (by 2))

(4) each point of $\Omega_{i-1} \cap$ bdy $\Delta_{i}$ has an open neighborhood disjoint from $\bigcup_{j>0} \bar{W}_{j}$. Define a $C^{0} \operatorname{map} \eta_{j}: w_{j} \rightarrow U_{i}$ as follows: $\eta_{0}=\delta_{i-1} \mid W_{0}$, and for $j>0$, pick $x_{j} \in W_{j}$, and let $\eta_{j}(x) \equiv \delta_{i-1}\left(x_{j}\right)$. For $x \in$ int $\Delta_{i}$ let

$$
\delta_{i}(x)=\phi_{i}^{-1}\left(\sum_{j \geq 0} \psi_{j}(x) \phi_{i}\left(\eta_{j}(x)\right)\right),
$$

and otherwise let $\delta_{i}(x)=\delta_{i-1}(x)$. It follows from (3) that for $x \in \operatorname{int} \Delta_{i}$,

$$
\begin{aligned}
& \left\|\phi_{i}\left(\delta_{i}(x)\right)-\phi_{i}\left(\delta_{i-1}(x)\right)\right\| \\
& \quad \leq \sum_{j>0} \psi_{j}(x)\left\|\phi_{i}\left(\delta_{i-1}\left(x_{j}\right)\right)-\phi_{i}\left(\delta_{i-1}(x)\right)\right\| \\
& \quad \leq \sum_{j>0} \psi_{j}(x) \tau_{i}(x) \leq \tau_{i}(x),
\end{aligned}
$$


and by (1) $d\left(\delta_{i}(x), \delta_{i-1}(x)\right) \leq \rho_{i}(x)$ for all $x \in \Delta$. From (4) each point of $\Omega_{i-1} \cap$ bdy $\Delta_{i}$ has an open neighborhood on which $\delta_{i}$ agrees with $\delta_{i-1}$, so (by the inductive hypothesis) $\delta_{i}$ is $C^{r}$ on $\Omega_{i}$, and $\delta_{i}$ has the desired properties.

4. Proof of TheOREM 1. According to $[\mathbf{Q}$, p. 215, Theorem 4] and [BS, p. 6, Theorem 1], if $M$ is a separable finite dimensional manifold and $r>(\operatorname{dim} M)+\nu$, then the maps transversal to $A$ are dense in $C^{r}(M, Y)$ in the fine $C^{r}$ topology [Mu, p. 29 and p. 32]. (In [Q, p. 215] the calculations show that $\operatorname{index}\left(\pi_{A} \circ h\right)=$ $\operatorname{dim} M+\operatorname{index} F$, not $\operatorname{dim} M-\operatorname{index} F$ as stated, and the corresponding change must be made in [Q, p. 215, Theorem 4].) Thus (1) if $\gamma: \Delta \rightarrow Y$ is the $C^{0}$ map of Lemma 3, we may suppose that the approximation $\delta$ is $C^{r}$ transversal to the map $A$ at each point of $\Delta-\Gamma$.

Let $(\Delta, \Gamma)$ be a finite polyhedral pair, and let $\gamma: \Delta \rightarrow Y$ and $\sigma: \Delta \rightarrow[0, \infty)$ be $C^{0}$ maps with $\sigma(x)=0$ if and only if $x \in \Gamma$. The Banach manifold $Y$ is an ANR [E, p. 766 or H-2, p. 95 ; p. 98, Theorem 8.1 ; and p. 96, Corollary 6.4 and Corollary 6.5]. From [H-2, p. 112, Theorem 1.2] there exists a $C^{0} \operatorname{map} \rho: \Delta \rightarrow[0, \infty)$ with $\rho(x)=0$ if and only if $x \in \Gamma$ such that, if $\delta: \Delta \rightarrow Y$ is $C^{0}$ and $d(\gamma(x), \delta(x)) \leq \rho(x)$, then there is a $C^{0}$ homotopy $h_{t}: \Delta \rightarrow[0, \infty)$ satisfying $h_{0}(x)=\gamma(x), h_{1}(x)=\delta(x)$, and $d\left(\gamma(x), h_{t}(x)\right) \leq \sigma(x)$.

Let $\gamma=\left(S^{m}, s_{0}\right) \rightarrow\left(Y, y_{0}\right)$, let $(\Delta, \Gamma)=\left(S^{m}, s_{0}\right)$, and let $\rho$ be given above (for any $\sigma)$. Let $\delta$ be given by (1) and Lemma 3 for this $\rho$ if $r>\max (m+\nu, 0)$; if $m+1 \leq k$, then $\gamma\left(S^{m}-s_{0}\right) \cap A\left(T_{k}(A)\right)=\varnothing$, so that $\gamma$ represents an element of $\pi_{m}\left(Y-A\left(T_{k}(A)\right), y_{0}\right)$ as desired.

Let $\gamma:\left(D^{m+1}, S^{m}, s_{0}\right) \rightarrow\left(Y, Y-A\left(T_{k}(A)\right), y_{0}\right)$, let $(\Delta, \Gamma)=\left(D^{m+1}, S^{m}\right)$, let $\rho$ be given for any $\sigma$, and let $\delta$ be given by (1) and Lemma 3 if $r>\max (m+1+\nu, 0)$; if $m+2 \leq k$, then $\gamma\left(D^{m+1}-S^{m}\right) \cap A\left(T_{k}(A)\right)=\varnothing$, so that $\gamma$ represents the trivial element of $\pi_{m}\left(Y-A\left(T_{k}(A)\right), y_{0}\right)$.

The proof shows more than is stated: any $\gamma:\left(S^{m}, s_{0}\right) \rightarrow\left(Y, y_{0}\right)$ with $y_{0} \notin$ $A\left(T_{k}(A)\right)$ may be approximated by a homotopic $\delta:\left(S^{m}, s_{0}\right) \rightarrow\left(Y-A\left(T_{k}(A)\right), y_{0}\right)$, and if $\delta$ is homotopic to the constant $y_{0}$ in $\left(Y, y_{0}\right)$, that homotopy may be approximated by a homotopy in $\left(Y-A\left(T_{k}(A)\right), y_{0}\right)$.

EXAMPLE 5. The differentiability hypotheses in Theorem 1 are sharp for all integers $m \geq 0, \nu$ and (a) $k=m+2[$ resp., (b) $k=m+1]$, i.e., injectivity in (a) and surjectivity in (b) are false if $C^{r}$ is replaced by $C^{r-1}$.

Let $P(s)$ be the statement: if $u>s \quad(s, u=1,2, \ldots)$, then there exists a $C^{u-s}$ map $h: \mathbb{R}^{u} \rightarrow \mathbb{R}^{s}$ such that $h\left(R_{0}(h)\right)=\mathbb{R}^{s}$, where $R_{0}(h)=T_{s}(h)$ is the set of points in $\mathbb{R}^{u}$ at which $D h$ has rank 0. According to [Whi], $P(1)$ is true (see also $[\mathbf{R}])$. The authors have not constructed examples to show that $P(s)$ is true for $s>1$, although it seems plausible that a generalization of Whitney's construction might be successful. We prove below:

If $P(s)$ is true for a given $s$ then for every integer $m \geq 0, \nu$, and (a) $k=$ $s+m+1$ [resp., (b) $k=s+m$ ], the differentiability hypothesis in Theorem 1 is sharp. Thus Example 5 results, and, if $P(s)$ is true for all positive integers $s$, then the differentiability hypothesis in Theorem 1 is sharp for all $m, \nu$ and $k$ (a) $m+2 \leq k$ [resp., (b) $m+1 \leq k]$.

PROOF. The differentiability hypothesis is (a) $r=1+\max (\nu+m+1,0)$ [resp., (b) $r=1+\max (\nu+m, 0)]$. If $r=1$, it is certainly sharp, so we may suppose that (a) $\nu+m \geq 0$ [resp., (b) $\nu+m \geq 1$ ]. Given any integers $m \geq 0, \nu$, and 
(a) $k \geq m+2$ [resp., (b) $k \geq m+1$ ], let (a) $s=k-m-1$ [resp., (b) $s=k-m$ ], let $u=k+\nu$, let $h: \mathbb{R}^{u} \rightarrow \mathbb{R}^{s}$ be the (a) $C^{\nu+m+1}$ [resp., (b) $C^{\nu+m}$ ] map given by $P(s)$, and define $\alpha: \mathbb{R}^{s} \rightarrow \mathbb{R}^{k}=\mathbb{R}^{s} \times \mathbb{R}^{k-s}$ by $\alpha(x)=(x, 0)$. Let $A=\alpha h$, so that $A\left(T_{k}(A)\right)=\alpha\left(\mathbb{R}^{s}\right)$; in case (a) $\pi_{m}\left(\mathbb{R}^{k}-A\left(T_{k}(A)\right) \neq 0\right.$ as desired.

In case (b) let $\Gamma=\left\{x \in \mathbb{R}^{m+1}: 1<\|x\|<2\right\}$ and let $V=\mathbb{R}^{s-1} \times \Gamma \subset \mathbb{R}^{k}$. Then $V$ and $V-\alpha\left(\mathbb{R}^{s}\right)$ have the homotopy types of $S^{m}$ and $S^{m-1}$, respectively $\left(S^{-1}=\varnothing\right)$. Let $U=A^{-1}(V)$, and let $B: U \rightarrow V$ be the restriction of $A$; then $B\left(T_{k}(B)\right)=\alpha\left(\mathbb{R}^{s}\right) \cap V$, and $B$ is the desired example for (b). [Actually example $B$ for (b) is also an example for (a): given an $m \neq 0$ for (b), $m-1$ is an $m$ for (a).]

These examples $A, B: \mathbb{R}^{n} \rightarrow \mathbb{R}^{p}$ have minimal $p(p=k)$, and other examples are $A \times \operatorname{id}_{E}$ and $B \times \operatorname{id}_{E}$, where $\mathrm{id}_{E}$ is the identity on a Banach space $E$. Some related examples are given in $[\mathbf{C - 3}$, p. 421, (3.3)] for case (a), and in [Sa, p. 890] for case (b) with $m=0$.

Proposition 6 [C-4, p. 1037, Proposition 4]. If $A: M^{n} \rightarrow N^{p}$ is a $C^{r}$ map on $C^{r}$ manifolds with $r \geq \max (n-p+k, 1)$, then $\operatorname{dim}\left(A\left(T_{k}(f)\right)\right) \leq p-k$.

EXAMPLE 7. For all positive integers $n, p$, and $k(k \leq p)$, the differential hypotheses in Proposition 6 are sharp.

PROOF. If $r=1$, the hypothesis is sharp, so we may suppose that $n-p+k \geq 2$. Let $u=n-p+k$, let $h: \mathbb{R}^{u} \rightarrow \mathbb{R}$ be the $C^{n-p+k-1}$ map of $P(1)$ in Example 5, define $g: \mathbb{R}^{n}=\mathbb{R}^{u} \times \mathbb{R}^{p-k} \rightarrow \mathbb{R} \times \mathbb{R}^{p-k}$ by $g(x, y)=(h(x), y)$, define $\alpha: \mathbb{R}^{1+p-k} \rightarrow$ $\mathbb{R}^{p}=\mathbb{R}^{1+p-k} \times \mathbb{R}^{k-1}$ by $\alpha(x)=(x, 0)$, and let $A=\alpha g$.

COROllaRY 8. Let $A: X \rightarrow Y$ be a $C^{r}$ proper Fredholm map of index $\nu$, where $r \geq \max (\nu+3,1)$ and $X$ and $Y$ are $C^{r}$ connected Banach manifolds with $X$ separable. Suppose that (a) for every nonempty connected open set $U \subset X, U-S A$ is nonempty and connected, and (b) for every $x \in X$, codim Range $D A(x) \neq 1$.

(I) Then $\nu \geq 0$ and there are $s(s=1,2, \ldots)$ and a compact connected $C^{r} \nu$ manifold $F^{\nu}$ without boundary such that,

(i) for each $y \in Y-A(S A), A^{-1}(y)$ is homeomorphic to $s$ disjoint copies of $F^{\nu}$, and

(ii) for $y \in A(S A), A^{-1}(y)$ has at most $s$ components.

(II) If, in addition, (c) codim $D A(x) \neq 2$ for every $x \in X$, then there is a factorization $A=\Psi \Phi$, where

(i) $Z$ is a $C^{r}$ connected Banach manifold,

(ii) $\Psi: Z \rightarrow Y$ is a $C^{r} s$-to-1 $(s=1,2, \ldots)$ diffeo-covering map,

(iii) $\Phi: X \rightarrow Z$ is a $C^{r}$ Fredholm map of index $\nu$ and, for every $z \in Z$, the set of solutions $x$ of $\Phi(x)=z$ is a nonempty compact connected set. In particular, if $z \notin \Phi(S \Phi)$ and $\nu=0$, then there is precisely one solution.

(III) The factorization is unique in the following sense: If $A=\bar{\Psi} \bar{\Phi}$ is another factorization with intermediate manifold $\bar{Z}$, then there is a $C^{r}$ diffeomorphism $\alpha: Z \rightarrow \bar{Z}$ such that $\bar{\Phi}=\alpha \Phi$ and $\bar{\Psi}=\Psi \alpha^{-1}$.

PrOOF. Let $V$ be any connected open set in $Y$. By hypothesis (b) the singular set $S A$ is $T_{2}(A)$, the set of $x \in X$ such that codim Range $D A(x) \geq 2$, and by Theorem 1, $V-A(S A)$ is connected. Since $A$ is proper, $A(S A)$ is closed in $Y$ and

$$
B_{V}=A \mid\left[A^{-1}(V-A(S A))\right]: A^{-1}(V-A(S A)) \rightarrow V-A(S A)
$$


is a $C^{0}$ bundle map [CK, argument of p. 151] whose fiber has $s$ components $(s=$ $0,1, \ldots)$. From hypothesis (a) and Theorem 1 neither $S A$ nor $A^{-1}(A(S A))$ can locally separate $A^{-1}(V)$ at any point, so that (2) for each component $U_{i}$ of $A^{-1}(V)$, $U_{i}-A^{-1}(A(S A)) \neq \varnothing$ and is a component of $A^{-1}(V-A(S A))$. Since $A$ is proper, $A\left(U_{i}\right)$ is closed in $V$ and since (by (1) and (2)) $V-A(S A) \subset A\left(U_{i}\right)$, (3) $A\left(U_{i}\right)=V$. Thus (4) $\nu \geq 0, s$ is independent of the choice of $V$ (by (1)), $s>0$, and (5) $A^{-1}(V)$ has at most $s$ components. If $A^{-1}(y)$ has at least $t$ components, then there is an open ball neighborhood $V$ of $y$ such that $A^{-1}(V)$ has at least $t$ components. By (5), $t \leq s$, so that (6) for every $y \in Y, A^{-1}(y)$ has at most $s$ components, and if $y \notin A(S A), A^{-1}(y)$ has exactly $s$ components by (1).

From (2) domain $\left(B_{Y}\right)$ is connected, and (7) $B_{Y}$ has a factorization $\varsigma \eta$, where $\varsigma$ is a $C^{0}$ bundle map with connected fiber and $\varsigma$ is a $C^{0} s$-to-1 covering map. (This is the monotone-light factorization [Why, pp. 141-143] of the proper map A.) Conclusion (I) follows from (4), (6), and (7).

Now assume hypothesis (II)(c) and that $V$ is simply connected, so that (Theorem 1) $V-A(S A)$ is simply connected. For each component $U_{i}$ of $A^{-1}(V)$,

$$
B_{V} \mid\left[U_{i}-A^{-1}(A(S A))\right]: U_{i}-A^{-1}(A(S A)) \rightarrow V-A(S A)
$$

is a $C^{0}$ bundle map with connected domain by (2), and from the homotopy sequence of a fibering [H-1, p. 152] its fiber is connected. From (3) $A^{-1}(V)$ and $A^{-1}(y)$ for $y \in V$ each have precisely $s$ components. The factorization in the $C^{0}$ category is immediate, and $Z$ is given the $C^{r}$ structure induced from $Y$ by $\Psi$, so that $\Phi$ is $C^{r}$ and $\Psi$ is a $C^{r}$ local diffeomorphism and $s$-to-1 covering map. Conclusion (II) results and conclusion (III) (uniqueness) is proved as in [C-2, p. 385, (3.6)].

REMARK 9. Other factorization results, in finite dimensions, are given in [C-2] and [CT-1]. Hypothesis (a) is required [C-1, p. 707, Examples 11 and 12].

COROLlaRY 10. Let $A: X \rightarrow Y$ be a $C^{r}$ proper Fredholm map of index $\nu$, where $r \geq \max (\nu+3,1), X$ is a $C^{r}$ connected separable Banach manifold and $Y$ is a Banach space. Suppose that (a) for every nonempty connected open set $U \subset X$, $U-S A$ is nonempty and connected and (b) for every $x \in X$, codim Range $D A(x)$ is neither 1 nor 2 . Then (i) $\nu \geq 0$; (ii) for each $y \in Y$, the set of solutions $x$ of $A(x)=y$ is a nonempty compact connected set; (iii) in particular, if $\nu=0$ and $y \notin A(S A)$, then there is precisely one solution.

ProOF. Since $Y$ is simply connected, $\Psi$ in Corollary 8 is a diffeomorphism [Ma, p. 159, Theorem 6.6 or p. 160 , Exercise 6.1 ].

Church thanks Syracuse University for research leave during 1986-87 and the University of Alberta for its hospitality and support during that period.

\section{REFERENCES}

[BS] J. G. Borisovic and J. I. Sapronov, On some topological invariants of nonlinear Fredholm mappings, Soviet Math. Dokl. 12 (1971), 5-9.

[CK] J. Cheeger and J .M. Kister, Counting topological manifolds, Topology 9 (1970), 149-151.

[C-1] P. T. Church, Differentiable maps with nonnegative Jacobian, J. Math. Mech. 16 (1967), 703708.

[C-2] _ Factorization of differentiable maps with branch set dimension at most $n-3$, Trans. Amer. Math. Soc. 115 (1965), 370-387. 
[C-3] _ On points of Jacobian rank k, Trans. Amer. Math. Soc. 110 (1964), 413-423.

[C-4] _, On points of Jacobian rank k. II, Proc. Amer. Math. Soc. 16 (1965), 1035-1038.

[CT-1] P. T. Church and J. G. Timourian, Differentiable maps with small critical set or critical set image, Indiana Univ. Math. J. 27 (1978), 813-831.

[CT-2] _ , A nonlinear elliptic operator and its singular values, Pacific J. Math. (to appear).

[E] J. Eells, Jr., A setting for global analysis, Bull. Amer. Math. Soc. 72 (1966), 751-807.

[H-1] S. T. Hu, Homotopy theory, Academic Press, New York, 1959.

[H-2] — Theory of retracts, Wayne State Univ. Press, Detroit, 1965.

[K] I. Kupka, Counterexample to the Morse-Sard theorem in the case of infinite dimensional manifolds, Proc. Amer. Math. Soc. 16 (1965), 954-957.

[Ma] W. S. Massey, Algebraic topology: An introduction, Springer-Verlag, New York, 1967 (fourth corrected printing, 1977).

[Mi] A. M. Micheletti, About differentiable mappings with singularities between Banach spaces, Analisi Funzionale e Applicazioni, Boll. Un. Mat. Ital. Suppl. 1 (1980), 287-301.

[Mu] J. R. Munkres, Elementary differential topology, Ann. of Math. Studies, no. 54, Princeton Univ. Press, Princeton, N.J., 1966.

[Q] F. Quinn, Transversal approximation on Banach manifolds, Proc. Sympos. Pure Math., vol. 15, Amer. Math. Soc., Providence, R.I., 1970, pp. 213-222.

[R] G. de Rham, Sur quelques fonctions différentiables dont toutes les valeurs sont des valeurs critiques, Celebrazioni Archimedee del Sec. XX (Siracusa, 1961), Vol. II, Edizioni "Oderisi," Gubbio, 1962, pp. 61-65.

[Sa] A. Sard, The measure of the critical values of differentiable maps, Bull. Amer. Math. Soc. 48 (1942), 883-890.

[Sm] S. Smale, An infinite dimensional version of Sard's Theorem, Amer. J. Math. 87 (1965), 861867.

[Sp] E. H. Spanier, Algebriac topology, McGraw-Hill, New York, 1966.

[Whi] H. Whitney, A function not constant on a connected set of critical points, Duke Math. J. 1 (1935), 514-517.

[Why] G. T. Whyburn, Analytic topology, Amer. Math. Soc., Providence, R.I., 1942.

Department of Mathematics, Syracuse University, Syracuse, New York 13244-1150

Department of Mathematics, University of Alberta, Edmonton, Alberta, CANADA T6G 2G1 